\title{
SPL Limitation of Use Terminology
}

National Cancer Institute

\section{Source}

National Cancer Institute. SPL Limitation of Use Terminology. NCI Thesaurus. Code C54599.

Terminology used for representation of information on factors that could limit use of medical product for a specific indication in the framework of the Structured Product Labeling documentation. 